\title{
EFECTO DE LA RADIACIÓN GAMMA SOBRE LAS CARACTERÍSTICAS MICROBIOLÓGICAS, FÍSICO-QUÍMICAS Y EVALUACIÓN SENSORIAL EN PIMIENTA NEGRA MOLIDA (Piper nigrum)
}

\section{EFFECT OF GAMMA RADIATION ON THE MICROBIOLOGIGAL, PHYSICO-CHEMICAL CHARACTERISTICS AND SENSORY ANALYSIS OF BLACK PEPPER POWDER (Piper nigrum)}

\author{
Johnny Vargas R. ${ }^{1}$, Marco Linares A. ${ }^{1}$, Flor de María Quispe M. ${ }^{2}$ \\ ${ }^{1}$ Instituto Peruano de Energía Nuclear, \\ Av. Canadá 1470, Lima 41, Perú \\ ${ }^{2}$ Probuysa EIRL, \\ Jr. Libertad 455, Lima 17
}

\section{RESUMEN}

La pimienta es la más famosa de las especias, se utiliza para dar sabor a los alimentos. Es una de las especias con mayor población microbiana, por lo que es causante de enfermedades y de descomposición de los alimentos principalmente procesados. El objetivo del presente trabajo es dar a conocer el efecto de la radiación gamma sobre la población microbiana, las características físico-químicas y la evaluación sensorial en pimienta negra molida, tratada con diferentes dosis alternativas, con la finalidad de obtener la dosis mínima óptima que reduzca la carga microbiana a las especificaciones recomendadas, sin alterar significativamente las características físico-químicas y sensoriales. Muestras de $500 \mathrm{~g}$ fueron irradiadas en el equipo Gammacell 220 (0; 4; 6 y 8 kGy). La tasa de dosis inicial fue de $5.82 \mathrm{kGy} / \mathrm{h}$. Se realizaron los siguientes análisis: microbiológico (Aerobios mesófilos, anaerobios, enterobacterias, Salmonella/ Shigella, Coliformes totales, Escherichia coli, Staphylococcus aureus, Estreptococos grupo D Lancefield, Bacillus cereus, Clostridium sulfito reductor, mohos y levaduras). Físico-químico (cenizas totales, cenizas insolubles en $\mathrm{HCl}$, solubles en $\mathrm{HCl}$, extracto alcohólico, extracto etéreo y fibra cruda). La evaluación sensorial (color, olor y sabor mediante test descriptivo. Los métodos fueron los recomendados por la FDA, APHA, AOAC y las NTP. A las diferentes dosis ensayadas, no se encontraron diferencias en los análisis físico-químicos y sensoriales. La dosis mínima seleccionada fue de $8 \mathrm{kGy}$ que redujo la población de microorganismos aerobios mesófilos en 5 ciclos logarítmicos de $9,8 \times 10^{6}$ a $4,0 \times 10 \mathrm{UFC} / \mathrm{g}$. Dosis de $4 \mathrm{kGy}$ fue suficiente para reducir la población de hongos en 3 ciclos logarítmicos de $1,7 \times 10^{3}$ a $<10 \mathrm{UFC} / \mathrm{g}$, cumpliendo con las especificaciones requeridas.

Palabras clave: Radiación gamma, pimienta, población microbiana, dosis.

\section{ABSTRACT}

Pepper is the most famous of spices used to flavor foods. It is one of the crops with the highest microbial population, and it is causing disease and decay of mostly processed foods. The aim of this study was to determine the effect of gamma radiation on microbial population, the physicalchemical and sensory evaluation in ground black pepper, treated with different doses alternatives, in order to obtain the optimal minimal dose that reduces microbial load to the recommended specifications, without significantly altering the physical-chemical and sensory. $500 \mathrm{~g}$ samples were irradiated in the Gammacell 220 (0, 4, 6 and $8 \mathrm{kGy}$ ). The initial dose rate was $5.82 \mathrm{kGy} / \mathrm{h}$. Were performed the following analysis: microbiological (aerobic mesophiles, anaerobes, Enterobacteriaceae, Salmonella / Shigella, total coliforms, Escherichia coli, Staphylococcus 
aureus, Lancefield group D streptococci, Bacillus cereus, Clostridium sulfite reducer, molds and yeasts). Physico-chemical (total ash, ash insoluble in $\mathrm{HCl}, \mathrm{HCl}$-soluble extract, alcohol, ether extract and crude fiber. Sensory evaluation (color, odor and flavor by descriptive test). The methods were those recommended by the FDA, APHA, AOAC and NTP. At different doses tested, there were no differences in physical-chemical analysis and sensory. The minimum dose of $8 \mathrm{kGy}$ was selected that reduced the population of aerobic plate in 5 $\log$ units of $9,8 \times 10^{6}$ to $4.0 \times 10 \mathrm{UFC} / \mathrm{g}$, dose of $4 \mathrm{kGy}$ was sufficient to reduce the fungal population by $3 \log$ units of $1,7 \times 10^{3}$ to $<10 \mathrm{UFC} / \mathrm{g}$, according to the specifications. Keywords: Gamma radiation, pepper, microbial population, dose.

\section{INTRODUCCION}

Las especias son ingredientes vegetales con carácter aromático que se utiliza habitualmente en pequeñas cantidades para conferir determinados sabores, aromas y colores, poseen además aceites esenciales y oleorresinas.

Las especias contienen frecuentemente gran número de microorganismos causantes de descomposición o de enfermedades cuando se incorporan a los alimentos, la población microbiana puede sobrepasar el nivel de $10^{8} \mathrm{UFC} / \mathrm{g}$ (cien millones de unidades de microorganismos formadores de colonias). La carga de hongos puede llegar a veces a $10^{5}$ $10^{6} \mathrm{UFC} / \mathrm{g}[1]$

La pimienta es una de las especias más contaminadas por diversos microorganismos, desde la cosecha y procesamiento es susceptible a la contaminación de bacterias y mohos

Algunos de sus usos están dirigidos a la elaboración de embutidos, formulaciones a base de vegetales, productos deshidratados, sopas y salsas preparadas.

Se debe tener en cuenta que la pimienta en polvo también es utilizada como tal, sin ningún tratamiento térmico posterior, como es el caso en el consumo de ensaladas frescas, elaboradas con lechugas, tomates paltas, etc. o en el popular ceviche, lo cual es riesgoso para el consumidor.

La irradiación puede ser utilizada para corregir deficiencias de calidad. Deberá evitarse la excesiva contaminación con microorganismos e insectos antes de la irradiación.

Por otra parte, deberán tomarse las medidas necesarias para minimizar la contaminación cuando ésta sea severa e inevitable.

Cuando se efectúan pruebas mediante métodos apropiados de muestreo y análisis, las especias, hierbas y otros sazonadores vegetales no tratados, cosechados $y$ manipulados bajo adecuadas condiciones higiénicas deberán presentar:

No más de $10^{4}$ bacterias coliformes por $\mathrm{g}$. No más de $10^{5}$ propágulos de mohos por $\mathrm{g}$.

Los objetivos de la irradiación en pimienta molida fueron:

Reducir la población microbiana de microorganismos aerobios mesófilas, mohos y levaduras.

Reducir el número de bacterias patógenas sin alterar significativamente sus características físico-químicas y sensoriales.

No se utiliza la irradiación para la preservación de estos productos. La preservación se obtiene mediante el empaque, almacenamiento adecuado y secado apropiado.

\section{Características del producto a irradiar}

El proceso de irradiación se aplica a estos productos en las formas en las cuales normalmente se preparan para su uso y comercialización. Las consideraciones usuales tomadas en cuenta son la calidad y el uso de las buenas prácticas de producción. La irradiación puede ser utilizada para corregir deficiencias de calidad. Deberá evitarse la excesiva contaminación con microorganismos antes de la irradiación. [2]

Dosis

La unidad de dosis absorbida es el Gray (Gy), que viene a ser la energía absorbida de un Joule por Kg. de materia irradiada: Gy = Joule/ $\mathrm{kg}$

\section{METODO EXPERIMENTAL}

Las muestras corresponden a una industria nacional.

Los productos fueron irradiados en el Equipo de Irradiación Gammacell 220 previa dosimetría Fricke y de acuerdo a la densidad 
y geometría del producto nos permitió hallar la tasa de dosis, para calcular los tiempos de exposición a la radiación gamma, para evaluar las dosis seleccionadas.

Para la dosimetría se utilizó el método ASTM E 1026 - 1995 Practice for Using the Fricke Reference Standard Dosimetry System Los métodos empleados en los análisis FísicoQuímico y las especificaciones para pimienta negra molida fueron consultados de la AOAC y las Normas Técnicas Nacionales (1984).

Los métodos empleados en los análisis microbiológicos fueron realizados de acuerdo a lo que señala la FDA (6ta Edición).

La evaluación sensorial se realizó mediante la prueba de comparación que permite encontrar diferencias entre dos o más muestras cuando estas son presentadas a los panelistas, quienes dan sus respuestas o calificaciones a través de términos descriptivos.

Con el objeto de obtener una medición cuantitativa se puntuaron los resultados sobre una escala hedónica de 1 a 5 puntos, donde:

$\begin{array}{ll}\text { Excelente } & -5 \text { puntos } \\ \text { Bueno } & -4 \text { puntos } \\ \text { Satisfactorio } & -3 \text { puntos } \\ \text { Regular } & -2 \text { puntos } \\ \text { Malo } & -1 \text { punto }\end{array}$

Intervinieron en el desarrollo de esta prueba 5 panelistas.

\section{RESULTADOS Y DISCUSION}

Tabla 1. Análisis microbiológico en pimienta negra molida.

\begin{tabular}{lcccc}
\hline DETERMINACIONES & Control & $\mathbf{4 ~ k G y}$ & $\mathbf{6 ~ k G y}$ & $\mathbf{8 ~ k G y}$ \\
\hline R.Aerobios mesófilos UFC/g & $9,8 \times 10^{6}$ & $2,6 \times 10^{4}$ & $3,6 \times 10^{3}$ & 40 \\
Recuento de Hongos UFC/g & $1,7 \times 10^{3}$ & $<10$ & $<10$ & $<10$ \\
Recuento Levaduras UFC/g & $1,4 \times 10^{2}$ & $<10$ & $<10$ & $<10$ \\
R.Anaerobios UFC/g & $2,5 \times 0^{5}$ & $1,5 \times 0^{2}$ & 10 & $<10$ \\
Enterobacterias UFC/g & $1,4 \times 10^{2}$ & $<10$ & $<10$ & $<10$ \\
D. Salmonella/Shigella (25g) & Ausente & Ausente & Ausente & Ausente \\
Coliformes totales (NMP/g) & 46,0 & 2,3 & 0,36 & $<0,30$ \\
Detección E. coli (en 25g) & Ausente & Ausente & Ausente & Ausente \\
R Clostridium sulfito r UFC/g & $5,0 \times 10$ & $<10$ & $<10$ & $<10$ \\
Staphylococcus aureus en 1 g & Ausente & Ausente & Ausente & Ausente \\
Streptococos GD Lancefield & 240 & $<0,3$ & 0,3 & 0,3 \\
Bacillus cereus UFC/g & $<10$ & $<10$ & $<10$ & $<10$ \\
& & & & \\
\hline
\end{tabular}

Podemos apreciar en la tabla 1 que la pimienta negra molida presenta una elevada contaminación de aerobios mesófilos de $9,8 \times 10^{6}$ UFC/g y al aplicarse la dosis de 8 kGy se logra reducir 5 ciclos logarítmicos (40 UFC/g) en lo que respecta a los hongos, podemos notar que es suficiente una dosis de 4 kGy para reducir completamente la presencia de hongos en 3 ciclos logarítmicos ( $<10$ UFC/g).

También a dosis de 8kGy son eliminados microorganismos como levaduras, anaerobios, enterobacterias, Coliformes totales, Clostridium sulfito reductor y Streptococos.

En la muestra de pimienta negra molida no se encontró Salmionella/Shiguella, Escherichia coli, Staphylococcus aureus y Bacilus cereus.

Tabla 2. Análisis físico-químico en pimienta negra molida

\begin{tabular}{lcccc}
\hline DETERMINACIONES & Control & 4 kGy & 6kGy & 8kGy \\
\hline Cenizas totales (\%) & 4,26 & 4,45 & 4,30 & 4,56 \\
Cenizas insolubles HCl\% & 0,58 & 0,58 & 0,56 & 0,57 \\
Cenizas solubles HCl\% & 3,68 & 3,87 & 3,74 & 3,99 \\
Extracto alcohólico (\%) & 4,79 & 4,56 & 4,52 & 4,53 \\
Extracto etéreo total (\%) & 9,07 & 8,93 & 9,20 & 9,20 \\
Fibra cruda (\%) & 12,92 & 13,21 & 12,46 & 13,68 \\
& & & & \\
\hline
\end{tabular}

Tabla 3. Especificaciones de análisis químico en pimienta negra [3]

\begin{tabular}{lcc}
\hline DETERMINACIÓN & ESPECIFICACIÓN & REFERENCIA \\
\hline Cenizas totales & Máximo $7.0 \%$ & N. ITINTEC 209.105 \\
Ceniza insoluble HCl & Máximo $1.5 \%$ & N. ITINTEC 209.105 \\
Extracto alcohólico & Mínimo $8.0 \%$ & N. ITINTEC 209.105 \\
Extracto etéreo & Máximo $7.0 \%$ & N. ITINTEC 209.105 \\
Fibra cruda & Máximo $15.0 \%$ & N. ITINTEC 209.105 \\
\hline
\end{tabular}

En lo que respecta a los análisis físicoquímicos no hay diferencias a las diferentes dosis ensayadas y están dentro de las especificaciones de la tabla 3 , excepto en el porcentaje de extracto alcohólico que exige un mínimo de $8 \%$ y los resultados de la investigación arrojan resultados entre $4,79 \%$ para el control y $4,53 \%$ para la dosis de $8 \mathrm{kGy}$, sin influir en estos datos las dosis administradas y en el análisis de extracto etéreo, la norma demanda un máximo de $7 \%$ y en las muestras ensayadas, para el control fue de $9,07 \%$ y a la dosis de 8 kGy $9,20 \%$, por lo que el efecto de la irradiación no tiene influencia, en esta comparación.

En los demás análisis realizados como en cenizas totales, no hay diferencias importantes, tenemos valores entre $4,26 \%$ a $4,56 \%$, siendo $7 \%$ el máximo requerido, al igual en cenizas insolubles en $\mathrm{HCl}$, para todos los tratamientos entre $0,56 \%$ y $0,58 \%$, siendo hasta $1.5 \%$ el aceptado.

También en fibra cruda se mantuvo constante entre 12,46 y 13,68 , debajo del máximo de $15 \%$. 
Tabla 4. Análisis sensorial en pimienta negra molida

\begin{tabular}{lcccc}
\hline Atributo & Control & $\mathbf{4 k G y}$ & 6kGy & 8kGy \\
\hline Color & Cenizo claro & Cenizo claro & Cenizo claro & Cenizo claro \\
Olor & $\begin{array}{c}\text { Aromático } \\
\text { punzante }\end{array}$ & $\begin{array}{c}\text { Aromático } \\
\text { punzante }\end{array}$ & $\begin{array}{c}\text { Aromático } \\
\text { punzante. }\end{array}$ & $\begin{array}{c}\text { Aromático } \\
\text { punzante }\end{array}$ \\
Sabor & Picante & Picante & Picante & Picante \\
Califica & Bueno & Bueno & Bueno & Bueno \\
Puntaje & 4 & 4 & 4 & 4 \\
\hline
\end{tabular}

En lo que respecta al análisis sensorial no se encontró diferencia entre el control y las diferentes dosis ensayadas, al evaluarse cuantitativamente, los jueces panelistas calificaron con un promedio de puntaje de 4 sobre una escala hedónica de 1 a 5 , correspondiendo a este puntaje una calificación de BUENO para todos los tratamientos.

\section{CONCLUSIONES}

La muestra presentó una calidad microbiológica deficiente con predominio de microorganismos aerobios mesófilos de $9,8 \times 10^{6} \mathrm{UFC} / \mathrm{g}$ y recuento de hongos de $1,7 \times 10^{3} \mathrm{UFC} / \mathrm{g}$.

No se encontraron Coliformes totales y Clostridium sulfito reductor.

La dosis mínima óptima de irradiación seleccionada fue de $8 \mathrm{kGy}$.

Dosis de 8kGy en pimienta negra molida reduce la población de microorganismos de aerobios mesófilos en 5 ciclos logarítmicos, cumpliendo con las especificaciones nacionales.
Dosis de 4kGy son suficiente para reducir la población de hongos en 3 ciclos logarítmicos de $1,7 \times 10^{3}$ UFC/g a $<10$ UFC/g.

A dosis de 8kGy las características físicoquímicas y sensoriales no son afectadas significativamente.

\section{REFERENCIAS}

[1] Farkas, J. Tratamiento de las Especias por Irradiación. La Irradiación de Alimentos en Latinoamérica IAEA TECDOC 331 Viena. (1985) 123-143

[2] Documento GCIIA N 5(1991). Código de la buena practicas de irradiación para el control de patógeno y otra microflora en especias, hierbas y otros sazonadores Vegetales. FAO/OIEA/OMS, Viena.

[3] Norma ITINTEC 209,105 Pimienta (1984)

E-mail: jvargas@ipen.gob.pe 